\title{
ANÁLISE DE MÉTODO ALTERNATIVO PARA PESQUISA DE ENTEROBIOSE
}

\section{ANALYSIS OF AN ALTERNATIVE METHOD TO RESEARCH ENTEROBIOSIS}

\author{
Eluize Carolina Teixeira ${ }^{1}$; Rosilda Aparecida Kovaliczn ${ }^{2}$; Priscilla Salles de Brito ${ }^{3}$ \\ ${ }^{1}$ Acadêmica do Curso de Farmácia na Universidade Estadual de Ponta Grossa (UEPG), Ponta \\ Grossa, PR. E-mail: <eluizect@hotmail.com>. \\ ${ }^{2}$ Professora da Disciplina de Parasitologia Humana na Universidade Estadual de Ponta Grossa \\ (UEPG) , Ponta Grossa. PR. E-mail: <rosildak@uol.com.br>. \\ ${ }^{3}$ Professora do Laboratório Escola na Universidade Estadual de Ponta Grossa- (UEPG), \\ Ponta Grossa, PR. E-mail: <priscillabrito@gmail.com>.
}

Data de recebimento: $12 / 11 / 2012$

Data da aprovação: 15/12/2012

\section{RESUMO}

As características biológicas da fêmea do Enterobius vermicularis Linnaeu, 1758, tornam a enterobiose bastante peculiar, já que raramente aparecem ovos do verme em exames coproparasitológicos de rotina e os métodos de diagnóstico na região perianal são invasivos e constrangedores. Com base na sintomatologia da parasitose, este estudo visa investigar a utilização de método menos invasivo para identificação de parasitados, utilizando-se material do leito subungueal. No período de outubro de 2011 a julho de 2012, foram investigadas 169 (100\%) crianças entre 02 e 05 anos de idade de seis Centros Municipais de Educação Infantil (CMEI) do bairro de Uvaranas - Ponta Grossa, PR. Com auxílio de uma espátula de metal higienizada, coletou-se o raspado do leito subungueal de cada um dos dedos das mãos das crianças, em frasco próprio e com conservante para análise microscópica. Paralelamente, realizou-se análise macro e microscópica das amostras fecais por método de sedimentação expontânea e centrífugo-flutuação. Nenhuma fase evolutiva do E. vermicularis foi encontrado em ambas as amostras, porém a análise fecal revelou: 11(6,50\%) com Giardia lamblia, 11(6,50\%) casos de Entamoeba coli, 07(4,14\%) com Ascaris lumbricoides, 05(2,95\%) positivos para Trichuris trichiura, $05(2,95 \%)$ com Endolimax nana, $01(0,59 \%)$ caso de Entamoeba histolytica/dispar e $01(0,59 \%)$ de Hymenolepis nana. A análise do material subungueal apresentou 07(4,14\%) crianças com ovos de Ascaris lumbricoides e $01(0,59 \%)$ caso de cistos de Entamoeba coli sob as unhas. Mesmo não revelando formas evolutivas de E. vermicularis, os resultados indicam que o material subungueal pode ser um exame complementar para a pesquisa de enteroparasitos.

Palavras-chave: Enterobiose. Leito subungueal. Exame complementar. Escolares.

\begin{abstract}
The biological characteristics of the female Enterobius vermicularis (Linnaeu, 1758) make enterobiosis very peculiar as the parasite eggs rarely appear on routine coproparasitological exams, and the methods of perianal diagnosis are invasive and embarrassing. Based on the symptomathology of the parasitosis, this study aims to
\end{abstract}


investigate the use of a less invasive method of identification of parasites using nailbeds material. In the period of October 2011 to July 2012, 169 (100\%) children between 2 and 5 years old from the Municipal Childhood Education Centers (CMEI, Portuguese abbreviation) of the district of Uvaranas - Ponta Grossa, PR were examined. With the aid of a sanitized metal spatula, material from the scrapped nailbeds of each the children's fingers was collected and stored in bottles with preservative solution for microscopic analysis. In addition to this, macro and microscopic analysis of fecal samples by spontaneous sedimentation and centrifugal-flotation method were performed. No evolutionary phase of $\mathbf{E}$. vermicularis was found in neither of the samples, however, the fecal analysis revealed: 11 children $(6.50 \%)$ with Giardia lamblia, $11(6,50 \%)$ cases of Entamoeba coli, 07 children (4,14\%) with Ascaris lumbricoides, 05 children (2,95\%) positive for Trichuris trichiura, 05 children (2,95\%) with Endolimax nana, 01 (0,59\%) case of Entamoeba histolytica/dispar, and 01 case $(0,59 \%)$ of Hymenolepis nana. The analysis of the subungual material presented 07 (4.14\%) children with Ascaris lumbricoides and 01 (0.59\%) case of cysts of Entamoeba coli under fingernails. Even without revealing evolutionary forms of $\mathbf{E}$. vermicularis, the results indicate that the examination of the subungual material can be a complementary test for the detection of intestinal parasites.

Keywords: Enterobiosis. Nail beds. Complementary exam. School children.

As parasitoses intestinais possuem importância para a saúde pública e o controle dessas infecções inclui educação sanitária, boas práticas de higiene, acompanhamento clínico e exames parasitológicos (BASSO et al., 2008).

A oxiurose ou enterobiose causada pelo Enterobius vermicularis Linnaeu, 1758, é uma enteroparasitose bastante peculiar devido às características biológicas da fêmea e dificilmente são encontrados ovos do verme em exames coproparasitológicos de rotina. A transmissão é direta de pessoa a pessoa, sem a intervenção do solo, e não requer condições especiais de ambiente, clima e nível social (CIMERMAN; CIMERMAN, 2005). Os métodos específicos para seu diagnóstico e recomendados na literatura são invasivos e constrangedores, uma vez que a pesquisa é realizada na região perianal.

Estetrabalhoteveporobjetivodetectarapresença de ovos e/ou vermes adultos de E. vermicularis em escolares da educação infantil por intermédio de método menos constrangedor, alternativo, para a pesquisa de casos de oxiurose utilizandose do raspado do leito subungueal. Paralelamente realizaram-se exames coproparasitológicos com a finalidade de comparar os resultados encontrados em ambas as análises.

\section{Revisão da literatura}

A enterobiose é uma parasitose prevalente em regiões de clima frio e temperado, inclusive em países mais desenvolvidos. Ambientes coletivos fechados, poeira intradomiciliar, menor frequência de banho e repetição de roupas estão entre os fatores que facilitam a disseminação e endemia do parasito (REY, 2010).

Machos e fêmeas habitam o intestino grosso e têm uma sobrevida de dois meses. O macho fecunda a fêmea e é eliminado, raramente sendo visto (NEVES, 2010).

As fêmeas, ovovivíparas, depois de fecundadas migram até o esfíncter anal e serpenteando pela região perianal e perineal liberam seus ovos, geralmente por rotura do tegumento. Os ovos tornam-se infectantes em torno de quatro a seis horas no meio externo (MORAES, 2008).

Entre os mecanismos básicos de transmissão, o principal é a autoinfecção externa no qual o próprio indivíduo infectado leva os ovos da região perianal à boca, ato responsável pela cronicidade da verminose devido à contaminação das extremidades dos dedos e unhas. Medidas de higiene pessoal são fundamentais para a erradicação da parasitose: banho matinal, corte rente das unhas, higienização do leito subungueal, 
lavagem das mãos sempre após defecação e antes de qualquer alimentação (NEVES, 2010; REY, 2010).

A ação patogênica do parasito é mecânica e irritativa devido à migração da fêmea e movimentação na região perianal, fato que causa intenso prurido, irritação local, vermelhidão e lesões na mucosa anal - possíveis escoriações provocadas pelas unhas do próprio indivíduo infectado ao coçar a região -, sintoma que se manifesta mais intensamente no período noturno e, em alguns casos, pode provocar infecções secundárias (NEVES, 2010; MORAES, 2008; REY, 2010). Os indivíduos sintomáticos podem apresentar também diarréias muco purulentas, dores abdominais, nervosismo, insônia e ligeiro grau de anemia (NEVES, 2010).

Casos de enterobiose ectópica são descritos, alguns raros como a infecção intraperitoneal com lesões granulomatosas relatada por Gazvani; Emery (1996), presença do parasito na uretra de paciente adulto do sexo masculino (ZAHARIOU; KARAMOUTI; PAPAIOANNOU, 2007) e, mais comumente, formas evolutivas do parasito na mucosa vaginal e região do períneo (LÓPEZ-OLMOS; GASULL, 2011). Em Minas Gerais, Rezende Neto et al. (2009) descrevem um caso de apendicite aguda por E. vermicularis. A enterobiose tem sido descrita na literatura médica também como causa de infecção crônica no referido órgão. Também em Minas Gerais, na área rural, Santos et al. (2002) relatam caso de adolescente, sexo feminino, com 13 anos de idade apresentando inflamação granulomatosa necrosante no ovário e, após exame microscópico do material de biópsia, revelou a presença de formas adultas de fêmeas de E. vermicularis com útero repleto de ovos.

O exame coproparasitológico de rotina não é o mais é indicado devido à característica da fêmea, que não realiza ovoposição no lúmen intestinal. A literatura indica como melhores técnicas para a detecção do parasito, os métodos de Graham e do Swab anal (NEVES, 2010; REY, 2010). Ambos os métodos requerem o uso de dispositivos colocados por aposição na região perianal para a investigação de ovos ou vermes adultos do E.vermicularis.

Devido à característica peculiar da fêmea não ovipor no interior do intestino, a prevalência da parasitose é bastante variável, uma vez que o parasito é pouco mencionado nos inquéritos coproparasitológicos que não utilizam métodos específicos para o parasito. Estudos com métodos não específicos, como o de Oliveira et al. (2001), revelaram uma prevalência de 3,5\% para E. vermicularis no município de Cambé - PR, utilizando-se os métodos de Hoffman, Pons e Janer e de Faust e Cols.

Em uma população escolar rural em Quipile, Colômbia, utilizando-se a técnica de Graham, foi encontrada uma prevalência de 2,5\% (KNUDSON et al., 2001). Já o trabalho realizado com pré-escolares em San Félix, Venezuela, também utilizando Graham, porém associada à análise de material do leito subungueal, revelou uma prevalência de $45,8 \%$ para $\mathbf{E}$. vermicularis. Neste último método os pesquisadores não encontraram nenhuma forma evolutiva do parasito em estudo (REQUENA et al., 2007).

Pezzani et al. (2004) encontraram 41,42\% das crianças da cidade de General Mansilla, Argentina, infectadas por E. vermicularis aplicando a técnica de passagem de uma compressa de gase, previamente umedecida com água, na região perianal. Outro estudo realizado também na Argentina, na cidade de Neuquen, revelou $46 \%$ de casos positivos aplicando-se os métodos de Telemann modificado e de Graham (BARBIERI et al., 2001).

No Brasil, em Belém - PA foram relatados $36,7 \%$ de positividade para enterobiose, utilizando-se a técnica de Graham modificada por Jacobs (1942) (COSTA, 1955).

O método de Ritchie (RITCHIE, 1948), empregado na análise do conteúdo subungueal, em Uberlândia - MG, não proporcionou dados de prevalência de E. vermicularis, sendo observados apenas ovos de Hymenolepis nana. (PEGHINI et al., 2001). Porém, em estudo realizado em Alicante, Espanha, utilizando a mesma técnica modificada com formol-etil-acetato na análise do material do leito subungueal, em associação com método de Graham, demonstrou uma prevalência de 4,3\% de E. vermicularis (MUÑOS et al., 2001). Observa-se que diferentes métodos têm sido utilizados em pesquisas com o intuito de evidenciar o parasitismo pelo E. vermicularis.

\section{Materiais e métodos}

No período de outubro de 2011 a julho de 2012, foram investigadas 169 crianças entre 02 e 05 anos de idade de seis Centros Municipais de Educação Infantil (CMEI) do bairro de Uvaranas - Ponta Grossa, 
PR. A pesquisa foi aprovada pelo Comitê de Ética e Pesquisa da UEPG, parecer $\mathrm{n}^{\circ} 100 / 2011$.

Primeiramente realizou-se uma reunião com pais/ responsáveis e professores para apresentação do projeto, procedimentos a serem efetuados, assinatura de termo autorizando a participação do escolar na pesquisa, distribuição gratuita de frasco coletor de fezes e agendamento da data para a entrega das amostras e coleta do material do leito subungueal das crianças.

Com a orientação para que as crianças não realizassem a lavagem prévia das mãos, o material do leito subungueal das unhas das mãos dos alunos participantes foi coletado no período da manhã e da tarde, no momento da sua chegada à escola, com o auxílio de uma espátula de inox pré-esterelizada, a qual foi passada no leito subungueal de todos os dedos das mãos, em processo cuidadoso, delicado, de raspagem para não ferir os dedos das crianças. A espátula era sempre desinfetada com álcool $70 \%$ antes de se iniciar a próxima coleta.

O material coletado foi armazenado em recipiente próprio, frasco de vidro com tampa de rosca e capacidade para $3 \mathrm{~mL}$, contendo $1 \mathrm{~mL}$ de solução conservante SAF (ácido acético, acetato de sódio e formol) devidamente identificados com os dados do aluno.

Os recipientes contendo as amostras do leito subungueal eram encaminhados de imediato para os laboratórios de Parasitologia da UEPG, permanecendo em repouso 24 horas para a sedimentação espontânea. Após este período, uma gota do sedimento foi coletada com auxílio de pipeta automática, depositado em lâmina de microscopia, e acrescido uma gota de lugol. Cada amostra foi observada em microscópio ótico em 100x e 400x.

Os exames de fezes foram executados por meio de dois métodos de enriquecimento: um de centrífugo-flutuação - Faust e Cols - para pesquisa de cistos e ovos leves, e um de sedimentação espontânea - Hoffman, Pons e Janer -, para cistos, ovos e larvas. Os laudos dos exames foram encaminhados para a direção da escola e, por seu intermédio, para os responsáveis pelos alunos, com a orientação para procura por tratamento médico dos casos positivos patogênicos.

Os resultados obtidos foram analisados estatisticamente através de análise de variância (ANOVA) de uma via, no nível de significância de $5 \%(\alpha=0,05)$.

\section{Resultados}

Das $169(100 \%)$ crianças que forneceram as amostras (fezes e amostra subungueal), 31(18,34\%) delas apresentaram positividade para enteroparasitos em pelo menos uma das amostras analisadas, sendo $24(14,20 \%)$ amostras positivas para fezes e 7 $(4,14 \%)$ positivas para material subungueal.

Observou-se na análise da matéria fecal que $11(6,50 \%)$ dos escolares apresentaram positividade para Giardia lamblia, 11(6,50\%) com Entamoeba coli, 07(4,14\%) casos de Ascaris lumbricoides, 05(2,95\%) de Trichuris trichiura, e 05(2,95\%) de Endolimax nana. Observou-se, ainda, 01(0,59\%) caso de Entamoeba histolytica/dispar e $01(0,59 \%)$ de Hymenolepis nana.

$\mathrm{Na}$ observação do material subungueal encontraram-se 07(4,14\%) escolares com Ascaris lumbricoides e 01(0,59\%) caso de Entamoeba coli, ressaltando-se que aqueles que apresentaram positividade na amostra fecal não eram os mesmos que se apresentaram positivos na análise do material subungueal (Tabela 01).

Tabela 1 - Prevalência de enteroparasitos em uma população de escolares do município de Ponta Grossa - PR, analisando-se matéria fecal e de leito subungueal, no período de outubro de 2011 a julho de 2012.

\begin{tabular}{|c|c|c|c|c|}
\hline \multirow[t]{2}{*}{ PARASITO } & \multicolumn{2}{|c|}{ MATÉRIA FECAL } & \multicolumn{2}{|c|}{ LEITO SUBUNGUEAL } \\
\hline & $\left(\mathrm{N}^{\circ}\right)$ & $(\%)$ & $\left(\mathrm{N}^{\circ}\right)$ & $(\%)$ \\
\hline Giardia lamblia & 11 & 6,50 & - & - \\
\hline Entamoeba coli & 11 & 6,50 & 01 & 0,59 \\
\hline Ascaris lumbricoides & 07 & 4,14 & 07 & 4,14 \\
\hline Trichuris trichiura & 05 & 2,95 & - & - \\
\hline Endolimax nana & 05 & 2,95 & - & - \\
\hline Hymenolepis nana & 01 & 0,59 & - & - \\
\hline $\begin{array}{l}\text { Entamoeba histolytica/ } \\
\text { dispar }\end{array}$ & 01 & 0,59 & - & - \\
\hline
\end{tabular}

Fonte: As autoras

\section{Discussão}

Não foram encontradas formas evolutivas do parasito E. vermicularis em nenhuma amostra de ambos os materiais biológicos, o que pode ser influência de uma série de fatores, que abrange desde o método 
empregado e até mesmo a biologia do parasito em estudo. O método preconizado por Goulart et al. (1966) e por Campus (1974) recomenda a coleta do material existente no leito subungueal do paciente logo após o mesmo acordar e antes de realizar a higiene da manhã por causa da eliminação dos eventuais ovos presentes no leito subungueal. Todavia, na presente pesquisa, mesmo que as coletas fossem realizadas no momento da chegada da criança à escola no período da manhã e da tarde, a grande maioria delas apresentava-se, neste momento, com as unhas aparadas e mãos lavadas, embora com orientação prévia de que não o fizessem antes da coleta do material.

A ausência de E. vermicularis no material subungueal corrobora com os achados de Requena et al.(2007). Porém é importante ressaltar que, no período de execução da pesquisa, os seis CMEI amostrados apresentavam estrutura física e disciplinar satisfatórias e investidores na educação sanitária dos escolares. Observou-se que as crianças são instruídas a lavarem corretamente as mãos sempre que necessário e a manter as unhas bem aparadas, higienizadas e, ainda, estimuladas ao uso constante de álcool em gel.

A positividade no material do leito subungueal para ovos de Ascaris lumbricoides e cistos de Entamoeba coli pode ser justificada devido a uma elevada carga parasitária que acomete a criança, contaminação das mãos durante a higiene anal, ou ainda formas evolutivas dos parasitos presentes no solo do peridomicílio. É sabido que, na criança em contato direto das mãos com o solo contaminado, há uma provável contaminação do leito subungueal, provando ser este local um depósito em potencial de ovos e cistos de enteroparasitos. A fêmea do Ascaris lumbricoides ovipõe no lúmem intestinal aproximadamente $200 \mathrm{mil}$ ovos/dia, os quais são eliminados junto com as fezes (REY, 2010). Conforme o destino da matéria fecal do parasitado, poderá ocorrer a contaminação do peridomicílio, ou, ainda, devido à falta de saneamento básico. O leito subungueal contaminado provém da precariedade da higienização das mãos e unhas. São fatores que contribuem para a positividade do material subungueal de forma individual ou associada.

O fato dos casos positivos para o leito subungueal não coincidirem com os mesmos escolares que apresentaram positividade na matéria fecal poderá ser justificado por bons hábitos de educação e higiene dessas crianças não levarem as mãos à boca. Ou então pode ter ocorrida falha na evidenciação dos parasitos na amostra fecal coletada, uma vez que foi solicitada uma única amostra de fezes.

Com a finalidade de avaliar a existência de uma diferença estatisticamente significante na análise das fezes e do material subungueal, aplicou-se a análise de variância (ANOVA), com a qual se observa que não houve diferença entre o resultado do exame de fezes e o resultado do material subungueal ao nível de significância de $5 \%\left(\mathrm{~F}_{1,10}=3,498789, \mathrm{P}>0,05\right)$. Determinou-se, portanto, que não existe uma relação significativa entre as amostras, uma vez que o escolar pode apresentar-se positivo para enteroparasitos na amostra fecal e também para amostras obtidas do leito subungueal.

\section{Conclusão}

Os resultados obtidos no presente trabalho demonstram que não houve a presença de nenhuma forma evolutiva do E. vermicularis nas amostras analisadas (fecal e leito ungueal), resultado para o qual contribuem alguns fatores, entre eles a impossibilidade de coleta do material subungueal logo após a criança levantar-se e antes de iniciar a higiene matinal. Outro fator é que durante a pesquisa in loco no ambiente escolar observaram-se condições satisfatórias de infraestrutura e adequada conscientização dos escolares de boas práticas de higiene, aplicadas atualmente nas escolas investigadas.

São discutíveis possíveis alterações na metodologia empregada, entre elas a maior participação dos pais ou responsáveis colaborando para que a criança não faça a higiene das mãos antes do momento da coleta, bem como a adição de técnicas específicas para o parasito E. vermicularis, embora mais invasivas e constrangedoras, como é o caso das técnicas de coleta na região perianal e perineal, mas que oferecem maior positividade.

Mesmo não revelando formas evolutivas de E. vermicularis, a presença de ovos de Ascaris lumbricoides e de cistos de Entamoeba coli no material subungueal sugere que este pode ser um exame complementar para a pesquisa de enteroparasitos e que o local é um potencial transmissor de parasitos intestinais por via oral. 


\section{REFERÊNCIAS}

BARBIERI, L. M. et al. Presencia de parasitos en niños de un asentamiento precario de la ciudad de neuquen y su relacion con las caracteristicas socioculturales y ambientales. Jornal Brasileiro de Patologia, v.37, n.4, p.141, 2001.

BASSO, R. M. C. et al. Evolução da prevalência de parasitoses intestinais em escolares em Caxias do Sul, RS. Revista da Sociedade Brasileira de Medicina Tropical, v. 41, n.3, p. 263-268, mai./jun. 2008.

CAMPOS, C. A. M. O depósito subungueal como disseminador de enteroparasitos. Revista Sociedade Brasileira de Medicina Tropical, v.8, p.93-97, 1974.

CIMERMAN, S.; CIMERMAN, B. Enterobíase. Revista Panamericana de Infectologia, v.7, n.3, p. 27-30, set. 2005.

COSTA, O. R. Incidência de Enterobius vermicularis em 359 escolares de Belém, Pará. Revista do Serviço Especial de Saúde Pública, Rio de Janeiro, v.8, p.221-229, 1955.

GAZVANI, M. R.; EMERY, S. J. Intraperitoneal Enterobius vermicularis Infection: A case report. Infectious Diseases in Obstetrics and Gynecology, United Kingdom, v.4, p. 28-30, abr.1996.

GOULART, E. G. et al. Pesquisa de cistos e ovos de enteroparasitas do homem no depósito subungueal. Revista Brasileira de Medicina, São Paulo, v. 26, n. 1, p. 465-466, jan. / abr. 1966.

GRAHAM, C. F. A device for the diagnosis of Enterobius infection. The American Journal of Tropical Medicine and Hygiene, v. 21, p.159-161, 1941.

KNUDSON, A. et al. Frequency of E. vermicularis infection in rural schoolchildren in Quipile, Colombia. Revista de Salud Pública, v.5, 2001.

LÓPEZ-OLMOS J, GASULL J. Enterobius vermicularis (oxiuros) en la práctica ginecológica: clínica y citología. Experiencia de 3 casos. Clínica e Investigación em Ginecología y Obstetricia, v.38, n.5, p.197-201, 2011.

MORAES, R. G. et al. Parasitologia e micologia humana. 5.ed. Rio de Janeiro: Guanabara Koogan, 2008.

MUÑOS, M. J. M. et al. Prevalência de enteroparasitosis en la población infantil assistente a guarderias públicas de la ciudade de Alcoy (Alicante), España. Jornal Brasileiro de Patologia, v.37, n.4, p.144, 2001.

NEVES, D.P. Parasitologia humana. 11.ed. São Paulo: Atheneu, 2010.

OLIVEIRA, F. J. A. et al. Estudo da prevalência de enteroparasitoses em escolares no município de Cambé-PR. Jornal Brasileiro de Patologia, v.37, n.4, p.133, 2001.

PEGHINI, B. C. et al. Levantamento parasitológico de Ascaris lumbricoides em crianças em idades escolar da cidade de Uberlândia (MG) através de método coproparasitológico e conteúdo subungueal. Jornal Brasileiro de Patologia, v.37, n.4, p. 138, 2001.

PEZZANI, B. C. et al. Enterobius vermicularis infection among population of General Mansilla, Argentina. World Journal of Gastroenterology, v.10, 2004.

REQUENA, I. et al. Enterobius vermicularis en preescolares de un área suburbana en San Félix, estado Bolívar, Venezuela. Investigación Clínica, v.48, n.3, p. 277-286, 2007.

REY, L. Bases da parasitologia médica. 3.ed . Rio de Janeiro: Guanabara Koogan, 2010.

REZENDE NETO, J. B. et al. Apendicite aguda por Enterobius vermicularis: relato de caso e revisão da literatura. Revista Medica Minas Gerais, v.19, n. 2, p. 180183, 2009.

SANTOS, V. M. et al. Nódulo granulomatoso com Enterobius vermicularis em epíploon simulando metástase de câncer de ovário. Revista da Sociedade Brasileira de Medicina Tropical, v.35, n.2, p. 191-193, mar. / abr. 2002.

ZAHARIOU, A.; KARAMOUTI, M. ; PAPAIOANNOU, P. Enterobius vermicularis in the male urinary tract: a case report. Journal of Medical Case Reports, Greece, v.01, p.137, nov. 2007. 